\title{
A novel indication of thioredoxin-interacting protein as a tumor suppressor gene in malignant glioma
}

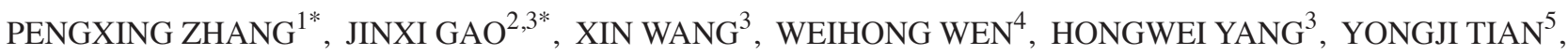 \\ NAN LIU ${ }^{1}$, ZHEN WANG ${ }^{1}$, HUI LIU ${ }^{1}$, YONGSHENG ZHANG ${ }^{1}$ and YANYANG TU ${ }^{1,3}$ \\ ${ }^{1}$ Department of Experimental Surgery, Tangdu Hospital, Fourth Military Medical University, Xi'an, Shaanxi 710038; \\ ${ }^{2}$ Department of Neurosurgery, Fuzhou General Hospital of The People's Liberation Army, Fuzhou, Fujian 350025, \\ P.R. China; ${ }^{3}$ Department of Neurosurgery, Brigham and Women's Hospital, Harvard Medical School, Boston, \\ MA 02115, USA; ${ }^{4}$ Department of Immunology, Fourth Military Medical University, Xi'an, Shaanxi 710032; \\ ${ }^{5}$ Department of Neurosurgery, Beijing Tiantan Hospital, Capital Medical University, Beijing 100050, P.R. China
}

Received May 9, 2016; Accepted April 13, 2017

DOI: $10.3892 / \mathrm{ol} .2017 .6397$

\begin{abstract}
Malignant glioma, the most common form of primary brain tumor, is associated with substantial morbidity and mortality, owing to the lack of response shown by patients to conventional therapies. Additional therapeutic targets and effective treatment options for these patients are therefore required. In the present study, a possible association of thioredoxin-interacting protein (TXNIP) with malignant glioma was evaluated. Initially, semi-quantitative and quantitative analysis of the expression levels of TXNIP in clinical specimens of primary glioma was performed via immunohistochemistry (IHC) and reverse transcription-quantitative polymerase chain reaction (RT-qPCR), respectively, and expression levels were further correlated to the overall survival time of the patients. The proliferative, migratory and invasive properties of the glioblastoma U251 cell line, engineered to downregulate TXNIP by lentiviral transfection of a specific short hairpin RNA, were evaluated by means of in vitro assays. Consequently, IHC and RT-qPCR analysis revealed a negative association between the expression level of TXNIP and the histopathological grade of the tumor. Higher TXNIP expression level was associated with extended patient survival time. In vitro analysis revealed increased growth, migration and invasion in U251 cells with downregulated TXNIP expression compared with their non-transfected counterparts. These findings strongly indicate that TXNIP functions as a tumor suppressor in malignant
\end{abstract}

Correspondence to: Professor Yanyang Tu or Professor Yongsheng Zhang, Department of Experimental Surgery, Tangdu Hospital, Fourth Military Medical University, 1 Xinsi Road, Xi'an, Shaanxi 710038, P.R China

E-mail: tu.fmmu@gmail.com

E-mail: zhangys_td@163.com

*Contributed equally

Key words: thioredoxin-interacting protein, short hairpin RNA, tumor suppressor gene, malignant glioma glioma cells and underscore its potential as a novel therapeutic target and prognostic indicator of the condition.

\section{Introduction}

Malignant glioma is an aggressive type of cancer and the most frequent form of primary brain tumor, which is mainly accompanied by a poor prognosis. Despite advanced therapeutic strategies, the median overall survival time of newly diagnosed glioblastoma patients remains at 1 to 2 years $(1,2)$. The primary treatment strategy for patients with malignant glioma consists of surgical resection followed by adjuvant radiation and chemotherapy. Therapeutic options for these patients are limited and the prognosis remains dismal $(3,4)$. Therefore, suppressing the aggressiveness of glioma cell proliferation and cell migration could be a novel and effective therapeutic strategy that deserves further research.

Thioredoxin-interacting protein (TXNIP), also known as vitamin D3 upregulating protein 1 or thioredoxin-binding protein 2 (5), is a multifunctional protein that is involved in cell proliferation, differentiation, and apoptosis $(6,7)$. TXNIP has been identified as a major redox regulator and a tumor suppressor gene in various solid tumors and hematological malignancies (8-10), but its role in malignant glioma cells or in glioma tissues has not been investigated prior to the present study.

\section{Materials and methods}

Patients and tissue samples. The study was approved by the Research Ethics Committee of Tangdu Hospital, the Fourth Military Medical University (Xi'an, China). All patients willingly consented to participate in the study by signing the written informed consent form. All specimens and data were handled according to the relevant ethical and legal standards.

A total of 54 glioma specimens (24 male and 30 female), resected between October 2008 and Octover 2010 and stored in liquid nitrogen, were retrieved from the archives of the Department of Pathology, Tangdu Hospital (Xi'an, China). The age of these patients ranged from 6 to 75 (mean age of 42.74 
and median age of 42.5 years). These retrieved specimens were directly used for reverse transcription- quantitative PCR (RT-qPCR) analysis, and formalin-fixed and paraffin-embedded for immunohistochemical (IHC) analysis. Conferring to the World Health Organization (WHO) classification (11), the histopathological sections were evaluated by two pathologists, with differences resolved by careful discussion. Accordingly, a total of 29/54 gliomas were classified as low-grade gliomas [7 pilocytic astrocytomas (WHO I) and 22 diffuse astrocytomas (WHO II)] and 25/54 as high-grade gliomas [20 anaplasia astrocytomas (WHO III) and 5 primary glioblastomas (WHO IV)]. None of the patients had been treated with chemotherapy or radiotherapy prior to surgery. The clinicopathological features of all patients are summarized in Table I.

The patient's survival time was assessed by calculating the days from the date of the initial glioma-related surgery to mortality. Patients who succumbed to diseases not directly associated with the gliomas or those who succumbed for other reasons were excluded from this study. The association between the overall survival time and the TXNIP expression level was established using the Kaplan-Meier method.

IHC analysis. Formalin-fixed, paraffin-embedded, sectioned tissues (4- $\mu \mathrm{m}$ thick) were stained using the Elivision Plus/horseradish peroxidase (HRP) detection system (Fuzhou Maixin Biotech Co., Ltd., Fuzhou, China) and counterstained with hematoxylin. In brief, following a peroxidase block with $3 \% \mathrm{H}_{2} \mathrm{O}_{2}$ /methanol (Fuzhou Maixin Biotech Co. Ltd.) for $30 \mathrm{~min}$, specimens were blocked with $5 \%$ normal goat serum. The slides were then incubated overnight with mouse polyclonal anti-human TXNIP primary antibody (cat. no. K0205-3; Medical \& Biological Laboratories Co., Ltd., Nagoya, Japan) at $1: 50$ dilution, at $4^{\circ} \mathrm{C}$. An IHC antibody diluent (cat. no. ZLI-9029; ZSGB-BIO, Beijing, China) at 1:50 dilution replaced the primary antibody for negative control slides. The specimens were then briefly washed three times with phosphate-buffered saline (PBS) and incubated at room temperature with Solution A for 20-min, followed by a 30-min incubation with Solution B (EnVision Plus/HRP mouse/Rabbit IHC kit; cat. no. KIT-9902; Fuzhou Maixin Biotech Co., Ltd.) at $37^{\circ} \mathrm{C}$. The signal visualization was performed by treatment with DAB chromogen for 2 to 3 min. After washing with water, specimens were counterstained with Meyer's hematoxylin (Maixin Biotech, Fuzhou, China). The WHO I neoplastic brain tissues of 54 glioma specimens, which were obtained from the archives of the Department of Pathology, Tangdu Hospital, were used as control tissues.

The evaluation standards of the pathologists were as follows: i) The intensity of the cell staining; non-staining as negative (-), light brown staining as weakly positive (+), brown staining as medium positive (++) and sepia staining as strong positive $(+++/++++)$; ii) the number of positive cells; the numbers of positive cells $<25 \%(+)$, the numbers of positive cells among $25-49 \%(++)$ and the numbers of positive cells $>50 \%$ (+++). Qualitative and half-quantitative staining results were achieved by combined the analysis results of the two experiments. A total of 5-10 high power fields were randomly observed and the averages were taken. Negative (-) and weakly positive (+) was regarded as low expression and the others were regarded as high expression.
Table I. Clinicopathological characteristics of glioma patients.

\begin{tabular}{lccc}
\hline & & \multicolumn{2}{c}{ TXNIP expression } \\
\cline { 4 - 4 } $\begin{array}{l}\text { Clinicopathological } \\
\text { features }\end{array}$ & $\begin{array}{c}\text { Patient } \\
\text { number }\end{array}$ & High, n (\%) & Low, n (\%) \\
\hline WHO grade & & & \\
I & 7 & $6(86)$ & $1(14)$ \\
II & 22 & $18(82)$ & $4(18)$ \\
III & 20 & $4(20)$ & $16(80)$ \\
IV & 5 & $0(0)$ & $5(100)$ \\
Age, years & & & $19(45)$ \\
$<55$ & 42 & $23(55)$ & $7(58)$ \\
$\geq 55$ & 12 & $5(42)$ & \\
Sex & & & $14(58)$ \\
Male & 24 & $10(42)$ & $12(40)$ \\
Female & 30 & $18(60)$ &
\end{tabular}

TXNIP, thioredoxin-interacting protein; WHO, World Health Organization.

Cell culture. The glioma U251MG cell line was purchased from the Chinese Academy of Sciences Cell Bank (Shanghai, China) and was stored in liquid nitrogen until use. The cell line was authenticated by short tandem repeat profiling. Under culture, the cells were maintained at $37^{\circ} \mathrm{C}$ in high-glucose Dulbecco's Modified Eagle's Medium (DMEM; Invitrogen; Thermo Fisher Scientific, Inc., Waltham, MA, USA) supplemented with $10 \%$ fetal bovine serum (Gibco; Thermo Fisher Scientific, Inc.) and $1 \%$ penicillin/streptomycin (Sigma-Aldrich; Merck KGaA, Darmstadt, Germany) in a humidified $95 \%$ air and $5 \% \mathrm{CO}_{2}$ incubator.

TXNIP downregulation and transfection. A total of 3 sequences of human lentiviral TXNIP short hairpin RNA (shRNA) (Table II) and the non-specific control shRNA were purchased and packaged into the lentivirus by Shanghai GenePharma Co., Ltd. (Shanghai, China). U251-MG cells were infected with $0.5 \mathrm{ml}$ shRNA lentiviruses $\left(1 \times 10^{6} / \mathrm{ml}\right)$ and cultured at $37^{\circ} \mathrm{C}$ in conventional DMEM supplemented with $2 \mu \mathrm{g} / \mathrm{ml}$ puro (Sigma-Aldrich; Merck KGaA) in a humidified $95 \%$ air and $5 \% \mathrm{CO}_{2}$ incubator for 1 week following transfection. These four cell lines were labeled as LV3-TXNIP shRNA1-U251, LV3-TXNIP shRNA2-U251, LV3-TXNIP shRNA3-U251 and LV3-NC-U251. The TXNIP expression level was evaluated by RT-qPCR and western blotting.

Western blot analysis and RT-qPCR analysis. For western blot analysis, cells (LV3-TXNIP shRNA1-U251, LV3-TXNIP shRNA2-U251, LV3-TXNIP shRNA3-U251 and LV3-NC-U251) were cultured to cell densities of $2-3 \times 10^{6}$ in $60 \mathrm{~mm}$ dishes. Subsequently, the cells were harvested and lysed with radio immunoprecipitation assay buffer (Beijing Solarbio Science \& Technology Co., Ltd., Beijing, China). Total protein concentrations were determined using the bicinchoninic acid protein assay kit (Boster Systems, Inc., Pleasanton, CA, USA). Total proteins $(20 \mu \mathrm{g})$ were divided by $12 \%$ SDS-PAGE (Genshare Biological, Shaanxi, China) 
Table II. Sequences of shRNAs and reverse transcription-quantitative polymerase chain reaction.

Identity

Sequence $\left(5^{\prime}-3^{\prime}\right)$

(Refs.)

TXNIP shRNA1

TXNIP ShRNA2

TXNIP shRNA3

TXNIP-qRT forward primer

TXNIP-qRT reverser primer

GAPDH forward primer

GAPDH reverse primer

ATCAGTCAGAGGCAATCATAT
GTGGAGGTGTGTGAAGTTA
CTCAAGACAGCCCTATCTTTA
TCATGGTGATGTTCAAGAAGATC
ACTTCACACACCTCCACTATC
GTATTGGGCGCCTGGTCAC
CTCCTGGAAGATGGTGATGG

ATCAGTCAGAGGCAATCATAT

CTCAAGACAGCCCTATCTTTA

TCATGGTGATGTTCAAGAAGATC

GTATTGGGCGCCTGGTCAC

CTCCTGGAAGATGGTGATGG

TXNIP, thioredoxin-interacting protein; qRT, quantitative reverse transcription; GAPDH, glyceraldehyde 3-phosphate dehydrogenase.

and transferred onto polyvinylidene fluoride membranes (EMD Millipore, Billerica, MA, USA). Following a 2-h blocking period with TBST buffer (TBS plus $0.1 \%$ Tween-20) supplemented with $5 \% \mathrm{w} / \mathrm{v}$ non-fat milk, the membranes were incubated overnight in TXNIP antibody (cat. no. K0205-3, Medical \& Biological Laboratories Co., Ltd.) at a 1:1,500 dilution at $4^{\circ} \mathrm{C}$. Subsequently, the membranes were incubated with HRP-conjugated goat anti-mouse secondary antibody (cat. no. BS50350, 1:5,000; Bioworld Technology, Inc., St. Louis Park, MN, USA) at room temperature for $1 \mathrm{~h}$. Following washing, proteins were visualized using an ECL detection system (Genshare Biological).

For RT-qPCR, RNA samples were extracted from the cells and 54 glioma tissues. Typically, $1 \mu \mathrm{g}$ total RNA was used to generate cDNA using SuperScript II RT (Takara Bio, Inc., Otsu, Japan) with an oligo-dT primer. RT-qPCR was performed using the Power SYBR-Green PCR Master mix, as per the manufacturer's instruction (Applied Biosystems; Thermo Fisher Scientific, Inc.). Thermo cycling for all reactions was initiated with a denaturation step at $95^{\circ} \mathrm{C}$ for $10 \mathrm{~min}$, followed by 40 cycles at $95^{\circ} \mathrm{C}$ for $5 \mathrm{sec}$ and $60^{\circ} \mathrm{C}$ for $30 \mathrm{sec}$. Each assay was performed in triplicate. GAPDH was used as the internal control. SDS 2.2.1 software (Applied Biosystems; Thermo Fisher Scientific, Inc.) was used to perform relative quantification of target genes using the comparative cycle threshold $\left(2^{-\Delta \Delta \mathrm{Cq}}\right)$ method (12). The primer sequences are described in Table II (13).

Cell proliferation assay. Cell proliferation was analyzed in vitro using tetrazolium salt 3-(4,5-dimethylthiazol-2-yl)-2,5diphenyltetrazolium bromide (MTT). A total of five identical 96-well plates were seeded simultaneously with the four types of cells aforementioned, at a concentration of $2 \times 10^{3}$ cells/well in $200 \mu \mathrm{l}$ DMEM. Each cell type was seeded into seven wells in each plate. After a designated culture time (12, 24, 48, 72 and $96 \mathrm{~h}$ ), the medium was replaced with $200 \mu \mathrm{l}$ medium supplemented with $0.5 \mathrm{mg} / \mathrm{ml}$ MTT (Sigma-Aldrich; KGaA) reagent and incubated at $37^{\circ} \mathrm{C}$ for $4 \mathrm{~h}$. Next, the supernatant was extracted, and the cells were lysed in $200 \mu \mathrm{l}$ dimethyl sulfoxide (Sigma-Aldrich; $\mathrm{KGaA}$ ) for $10 \mathrm{~min}$ at $37^{\circ} \mathrm{C}$. The optical density values were measured by recording the absorbance at $490 \mathrm{~nm}$ using a plate reader. The obtained values were presented as the fold increase with respect to the control group.
Wound-healing assays. U251-MG cells were seeded in 6-well plates and cultured until they reached confluence. A wound was then created by manually scraping the cell layer with a $200-\mu 1$ pipette tip. The floating cells were removed by washing with PBS three times. The cells were then incubated in DMEM supplemented with $1 \%$ FBS for $24 \mathrm{~h}$. For each group, the number of cells that had migrated into the simulated wound was counted by imaging eight randomly selected microscopic fields at 0,12 and $24 \mathrm{~h}$ after wound creation. Images were acquired with a Nikon DS-5 M Camera System (Nikon Corporation, Tokyo, Japan) mounted on a phase-contrast Leitz microscope (Leica Microsystems GmbH, Wetzlar, Germany) and were processed using Adobe Photoshop 7.0 (Adobe Systems, Inc., San Jose, CA, USA).

In vitro migration and invasion assays. Migration and invasion experiments were performed using a QCM 24-Well Cell Invasion assay kit (Cell Biolabs, Inc., San Diego, CA, USA). The top chamber of the Transwell system received $2 \times 10^{3}$ cells, plated directly on the polycarbonate Transwell filters (for the Transwell migration assay) or on the Matrigel-coated polycarbonate Transwell filter (for the Transwell matrix-penetration invasion assay). For the Transwell migration assay, the top (containing the cells) and bottom chambers received serum-free medium. However, for the invasion assay, cells in the top chamber were suspended in serum-free medium, whereas the bottom chamber received medium supplemented with $10 \%$ FBS, serving as a chemoattractant. The cells were incubated at $37^{\circ} \mathrm{C}$ for $8 \mathrm{~h}$ (for the migration assay) or $16 \mathrm{~h}$ (for the invasion assay). The non-migratory or non-invading cells on the upper surface of the polycarbonate membrane in the top chamber were wiped away using cotton swabs. The migrated and invaded cells on the lower surface of the membrane were fixed in $95 \%$ ethanol for $5 \mathrm{~min}$, air-dried and stained with $4 \mathrm{~g} / \mathrm{l}$ crystal violet prior to counting under a microscope. A total of three independent experiments were conducted and the data are presented as the mean \pm standard error of the mean (SEM).

Statistical analysis. All computations were performed using SPSS version 13.0 software, for Windows (SPSS, Inc., Chicago, IL, USA). Data are expressed as the mean \pm SEM throughout. Statistical differences between the levels of TXNIP expression in different pathological grades were evaluated with the non-parametric Kruskal-Wallis test, and those differences 

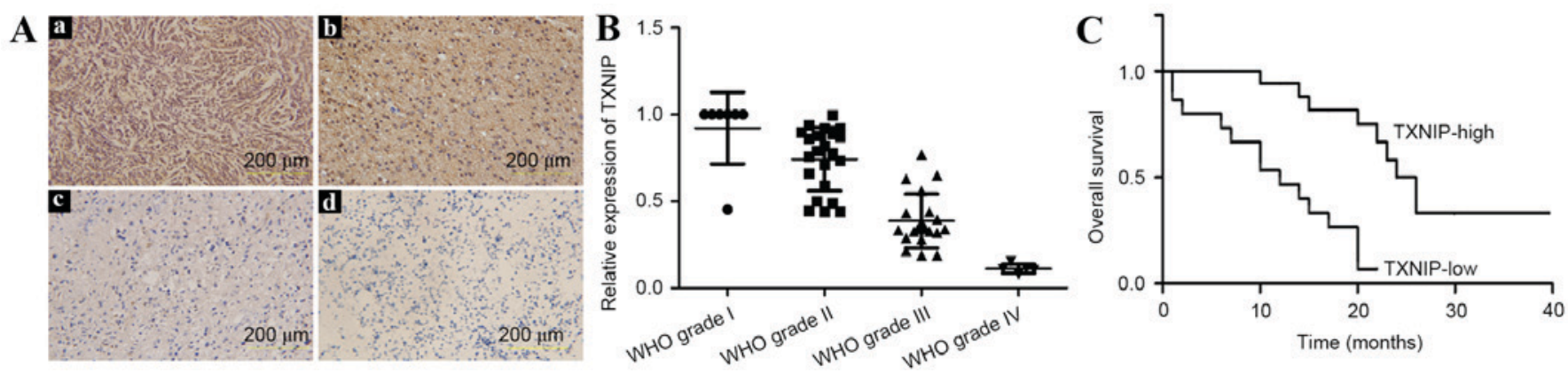

Figure 1. (A) Representative images of TXNIP immunohistochemical staining by treatment with DAB chromogen and hematoxylin in (A-a) WHO grade I gliomas, (A-b) WHO grade II gliomas, (A-c) WHO grade III gliomas and (A-d) WHO grade IV gliomas. The expression level of TXNIP was significantly decreased in higher grade tumors $(\mathrm{P}<0.05)$. (B) The relative expression of TXNIP mRNA detected by reverse transcription-quantitative polymerase chain reaction. The expression level of TXNIP mRNA was significantly decreased in higher grade tumors. (C) The association of TXNIP expression with the overall survival of glioma patients. WHO, World Health Organization; TXNIP, thioredoxin-interacting protein.

A

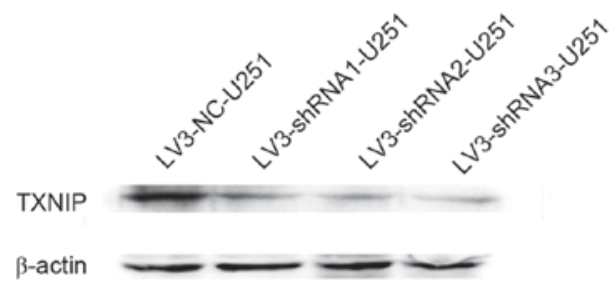

B

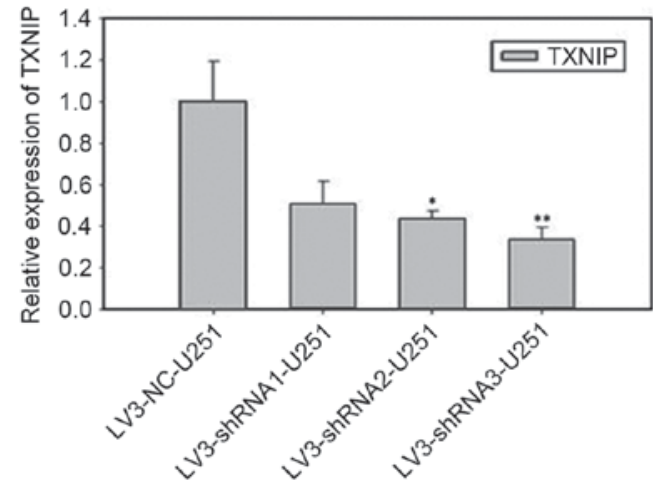

Figure 2. (A) Western blot analysis and (B) reverse transcription-quantitative polymerase chain reaction detected the expression of TXNIP in cells. Error bars represent standard error of the mean. ${ }^{*} \mathrm{P}<0.05,{ }^{* *} \mathrm{P}<0.01$. Scale bar, $50 \mathrm{~mm}$. TXNIP, thioredoxin-interacting protein. NC, negative control; shRNA, short hairpin RNA.

in the binominal clinical categories were evaluated with the non-parametric Mann-Whitney test. The survival time was calculated according to the Kaplan-Meier method. Differences between values obtained from in vitro experiments of the treatment groups were compared to the untreated control group by paired Student's t-test. $\mathrm{P}<0.05$ was considered to indicate statistical significance.

\section{Results}

Low expression of TXNIP in human glioma tissues. In the 54 patients with gliomas, IHC analysis revealed high TXNIP expression, localized mainly in the nucleus in 51.9\% (28/54) of samples (Fig. 1A), the majority of which were from low-grade glioma patients. The remaining 48.1\% (26/54) of samples demonstrated lower TXNIP expression, which was found to be associated with a higher histopathological glioma grade $(\mathrm{P}<0.01)$

To validate the specificity of the immunohistochemical results, RT-qPCR analysis was performed. The primers are listed in Table II. TXNIP was strongly expressed in low-grade glioma samples, but was not detected or was only weakly detected in high-grade gliomas, particularly in glioblastoma (grade IV) (Fig. 1B). These results were consistent with those of the IHC analysis.

To analyze the overall survival time, the patients were followed up for a total of 40 months from the initiation of the study, during which 52/54 patients succumbed to glioma. The Kaplan-Meier analysis showed a strong association of TXNIP expression with the overall survival of glioma patients (Fig. 1C); high expression was associated with increased overall survival time.

Construction and identification of TXNIP shRNA-stabilized cell line. U251-MG cells were transfected with one of the TXNIP shRNA lentiviruses (shRNA1-3) or the scrambled shRNA lentivirus. The results of the western blot analysis (Fig. 2A) and RT-qPCR (Fig. 2B) revealed that transfection of shRNA2 and shRNA3 was more efficient than shRNA1, and that shRNA3 transfection was more efficient $(\mathrm{P}<0.01)$ than shRNA2 transfection $(\mathrm{P}<0.05)$.

Downregulation of TXNIP promotes glioma cell invasion, migration and proliferation. Several studies have shown that TXNIP is strongly downregulated in several types of clinical tumor samples and tumor cell lines (10,14-16). It was hypothesized that the downregulation of TXNIP would induce a more aggressive behavior in cultured glioma cells. To test this hypothesis, the glioma U251-MG cell line was infected with a lentivirus encoding an shRNA sequence targeted at TXNIP. First, an MTT assay was used to examine the effect of TXNIP on cell proliferation. The cell proliferation was increased in the TXNIP shRNA group compared with the control groups at $24 \mathrm{~h}$ after transfection, indicating that downregulation of TXNIP 

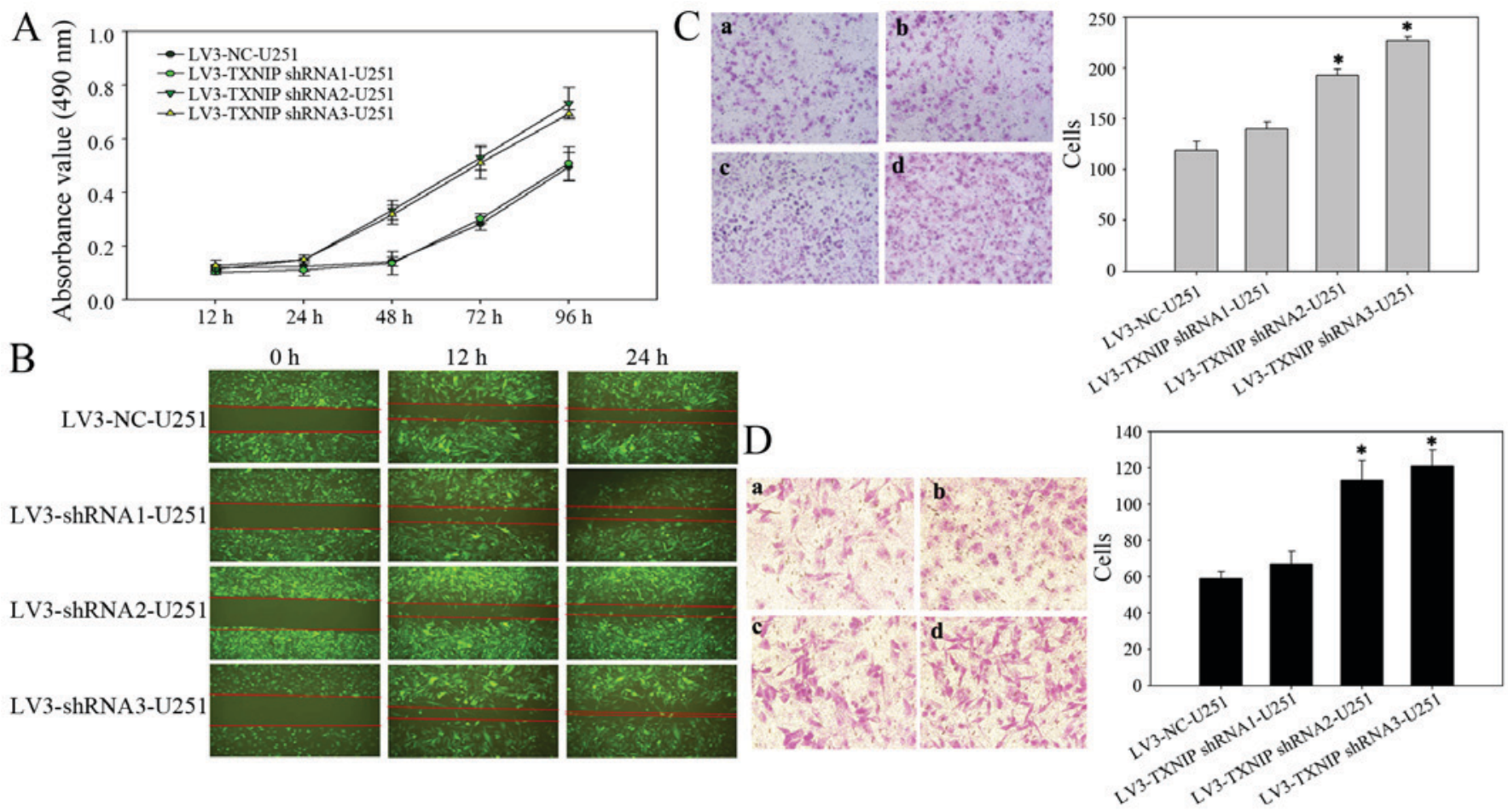

Figure 3. Downregulated expression of TXNIP promotes glioma cell invasion, migration and proliferation in vitro. (A) Growth curves following cell transfection with the indicated shRNA were assessed by 3-(4,5-dimethylthiazol-2-yl)-2,5- diphenyltetrazolium bromide cell proliferation assay. (B) The wound-healing assay revealed a difference in cell migratory abilities between LV3-NC-U251 and LV3-TXNIP shRNA-U251 cells. (C) Transwell invasion and (D) migration assay of TXNIP-transfected or untransfected U251-MG cells. (C-a, D-a) LV-NC-U251, (C-b and D-b) LV-TXNIP shRNA1-U251, (C-c and D-c) LV-TXNIP shRNA2-U251 and (C-d and D-d) LV-TXNIP shRNA3-U251. "P<0.05 vs. LV3-NC-U251 cells. TXNIP, thioredoxin-interacting protein; shRNA, short hairpin RNA; NC, negative control.

could significantly promote the proliferation of glioma cells (Fig. 3A). A wound-healing assay was then used to examine the effect of TXNIP on cell migration. Compared with the LV-NC cells, the TXNIP shRNA cells exhibited rapid migration (Fig. 3B). Furthermore, Transwell assays revealed that the downregulation of TXNIP significantly enhanced the invasion (Fig. 3C) and migration (Fig. 3D) of glioma cells.

\section{Discussion}

Cancer development is a highly-orchestrated process that requires complex transcriptional and post-transcriptional regulation of gene expression (17). TXNIP has recently been established as a tumor suppressor gene, the downregulation of which is associated with tumorigenesis and metastasis (8-10). Low expression of TXNIP has been observed in breast, liver, stomach and other cancer types (10,14-16). TXNIP deficiency may promote tumor cell proliferation and inhibit apoptosis $(9,10,18)$. In relation to its antitumor mechanisms, TXNIP is essential in a variety of normal cellular functions, including natural killer cell development, cellular growth inhibition and apoptosis, thioredoxin-mediated antioxidant function and the modulation of other molecules, including mediator complex subunit 23, KiSS-1 metastasis-suppressor and exportin $1(9,10,19-21)$.

Although TXNIP expression is frequently suppressed in human cancer, genetic alterations such as translocation, deletion or somatic point mutation are uncommon (22). This lack of genetic alteration suggests that aberrant TXNIP expression in cancer is mainly regulated via post-transcriptional and translational mechanisms. TXNIP promoter hypermethylation was first observed in renal cell carcinoma using methylation-specific PCR, induced by ferric nitrilotriacetate (23). Ahsan et al (24) proposed that DNA methylation could be implicated in the silencing of TXNIP expression in leukemia. The process of DNA methylation is an established method of silencing various other tumor suppressor genes (25-27). In addition to this, histone deacetylation, regulation by microRNAs and protein translation may also serve important roles in repressing TXNIP expression (14,28-30).

In the present study, IHC analysis of the expression level of TXNIP was conducted in surgically resected glioma tissues, which revealed the marked downregulation of TXNIP in high-grade human glioma compared with low-grade human glioma. Furthermore, under in vitro conditions, shRNAs were used to downregulate expression of TXNIP in the U251-MG glioma cell line, which enhanced the invasion, migration and proliferation of the glioma cells. Downregulation of TXNIP converted the cells to an aggressive cancer phenotype, which may be representative of cells in high-grade human glioma. The results of the present study established a strong inverse association between TXNIP expression level and the glioma aggressiveness and histopathological grading of the disease, and a direct association with patient survival time.

In conclusion, from the results of the present study it is apparent that the tumor suppressor activity of TXNIP is strongly associated with glioma development and progression. These data highlight the importance of TXNIP as a potential 
therapeutic target and prognostic marker in advanced human gliomas.

\section{Acknowledgements}

This study was supported by grants from the National Natural Scientific Foundation of China (nos. 81272419 and 81572983), the Social Development of Technology Research Projects in Shaanxi Province (no. 2015SF027), the Natural Scientific Foundation of Shaanxi Province (no. 2014JM4148) and the Beijing Key Laboratory of Brain Major Diseases Open Project (2015).

\section{References}

1. Chang L, Su J, Jia XZ and Ren H: Treating malignant glioma in Chinese patients: Update on temozolomide. Onco Targets Ther 7: 235-244, 2014.

2. Davis FG and McCarthy BJ: Current epidemiological trends and surveillance issues in brain tumors. Expert Rev Anticancer Ther 1: 395-401, 2001.

3. Yang P, Wang Y, Peng X, You G, Zhang W, Yan W, Bao Z, Wang Y, Qiu X and Jiang T: Management and survival rates in patients with glioma in China (2004-2010): A retrospective study from a single-institution. J Neurooncol 113: 259-266, 2013.

4. Chen C, Xu T, Lu Y, Chen J and Wu S: The efficacy of temozolomide for recurrent glioblastoma multiforme. Eur J Neurol 20: 223-230, 2013.

5. Chen KS and DeLuca HF: Isolation and characterization of a novel cDNA from HL-60 cells treated with 1, 25-dihydroxyvitamin D-3. Biochim Biophys Acta 1219: 26-32, 1994.

6. Kim SY, Suh HW, Chung JW, Yoon SR and Choi I: Diverse functions of VDUP1 in cell proliferation, differentiation, and diseases. Cell Mol Immunol 4: 345-351, 2007.

7. Patwari P, Chutkow WA, Cummings K, Verstraeten VL, Lammerding J, Schreiter ER and Lee RT: Thioredoxin-independent regulation of metabolism by the alpha-arrestin proteins. J Biol Chem 284: 24996-25003, 2009.

8. Zhou J, Yu Q and Chng WJ: Txnip (vdup-1,tbp-2): A major redox regulator commonly suppressed in cancer by epigenetic mechanisms. Int J Biochem Cell Biol 43: 1668-1673, 2011.

9. Zhou JB and Chng WJ: Roles of thioredoxin binding protein (TXNIP) in oxidative stress, apoptosis and cancer. Mitochondrion 13: 163-169, 2013.

10. Yoshihara E, Masaki S, Matsuo Y, Chen Z, Tian H and Yodoi J: Thioredoxin/Txnip: Redoxisome, as a redox switch for the pathogenesis of diseases. Front Immunol 4: 514, 2014.

11. Louis DN, Ohgaki H, Wiestler OD, Cavenee WK, Burger PC, Jouvet A, Scheithauer BW and Kleihues P: The 2007 WHO classification of tumours of the central nervous system. Acta Neuropathol 114: 97-109, 2007.

12. Livak KJ and Schmittgen TD: Analysis of relative gene expression data using real-time quantitative PCR and the 2(-Delta Delta C(T)) method. Methods 25: 402-408, 2001.

13. Zhou J, Bi C, Cheong LL, Mahara S, Liu SC, Tay KG, Koh TL, Yu Q and Chng WJ: The histone methyltransferase inhibitor, DZNep, up-regulates TXNIP, increases ROS production, and targets leukemia cells in AML. Blood 118: 2830-2839, 2011.

14. Butler LM, Zhou X, Xu WS, Scher HI, Rifkind RA, Marks PA and Richon VM: The histone deacetylase inhibitor SAHA arrests cancer cell growth, up-regulates thioredoxin-binding protein-2, and down-regulates thioredoxin. Proc Natl Acad Sci 99: 11700-11705, 2002.

15. Yamaguchi F, Takata M, Kamitori K, Nonaka M, Dong Y, Sui L and Tokuda M: Rare sugar d-allose induces specific up-regulation of txnip and subsequent G1 cell cycle arrest in hepatocellular carcinoma cells by stabilization of p27 $7^{\mathrm{kipl}}$. Int J Oncol 32: 377-385, 2008.
16. Song H, Cho D, Jeon JH, Han SH, Hur DY, Kim YS and Choi I: Vitamin d (3) up-regulating protein 1 (vdup1) antisense DNA regulates tumorigenicity and melanogenesis of murine melanoma cells via regulating the expression of fas ligand and reactive oxygen species. Immunol Lett 86: 235-247, 2003.

17. Ying Z, Li Y, Wu J,Zhu X, Yang Y, Tian H, Li W, Hu B, Cheng SY and $\mathrm{Li} \mathrm{M}$ : Loss of miR-204 expression enhances glioma migration and stem cell-like phenotype. Cancer Res 73: 990-999, 2013.

18. Zhang P, Pang X and Tu Y: Thioredoxin-interacting protein as a common regulation target for multiple drugs in clinical therapy/application. Cancer Transl Med 1: 26-30, 2015.

19. Han SH, Jeon JH, Ju HR, Jung U, Kim KY, Yoo HS, Lee YH, Song KS, Hwang HM, Na YS, et al: VDUP1 upregulated by TGF-beta1 and 1,25-dihydorxyvitamin D3 inhibits tumor cell growth by blocking cell-cycle progression. Oncogene 22: 4035-4046, 2003.

20. Le Jan S, Le Meur N, Cazes A, Philippe J, Le Cunff M, Léger J, Corvol P and Germain S: Characterization of the expression of the hypoxia-induced genes neuritin, txnip and IGFBP3 in cancer. FEBS Lett 580: 3395-3400, 2006.

21. Lee KN, Kang HS, Jeon JH, Kim EM, Yoon SR, Song H, Lyu CY, Piao ZH, Kim SU, Han YH, et al: Vdup1 is required for the development of natural killer cells. Immunity 22: 195-208, 2005.

22. Erkeland SJ, Palande KK, Valkhof M, Gits J, Danen-van Oorschot A and Touw IP: The gene encoding thioredoxin-interacting protein (TXNIP) is a frequent integration site in virus-induced mouse leukemia and is overexpressed in a subset of AML patients. Leuk Res 33: 1367-1371, 2009.

23. Dutta KK, Nishinaka Y, Masutani H, Akatsuka S, Aung TT, Shirase T, Lee WH, Yamada Y, Hiai H, Yodoi J and Toyokuni S: Two distinct mechanisms for loss of thioredoxin-binding protein-2 in oxidative stress-induced renal carcinogenesis. Lab Invest 85: 798-807, 2005.

24. Ahsan MK, Masutani H, Yamaguchi Y, Kim YC, Nosaka K, Matsuoka M, Nishinaka Y, Maeda M and Yodoi J: Loss of interleukin-2-dependency in HTLV-I-infected T cells on gene silencing of thioredoxin-binding protein-2. Oncogene 25: 2181-2191, 2006.

25. Cheng YD, Geng H, Cheng SH, Liang P, Bai Y, Li J, Srivastava G, Ng MH, Fukagawa T, Wu X, et al: The KRAB Zinc finger protein ZNF382 is a general, proapoptotic tumor suppressor repressing multiple oncogenes and frequently silenced in multiple carcinomas. Cancer Res 70: 6516-6526, 2010.

26. Cheng YD, Liang P, Geng H, Wang Z, Li L, Cheng SH, Ying J, $\mathrm{Su}$ X, Ng KM, Ng MH, et al: A novel 19q13 nucleolar zinc finger protein suppresses tumor cell growth through inhibiting ribosome biogenesis and inducing apoptosis but is frequently silenced in multiple carcinomas. Mol Cancer Res 10: 925-936, 2012.

27. Wang S, Cheng Y, Du W, Lu L, Zhou L, Wang H, Kang W, Li X, Tao Q, Sung J and Yu J: Zinc-finger protein 545 is a novel tumour suppressor that acts by inhibiting ribosomal RNA transcription in gastric cancer. Gut 62: 833-841, 2013.

28. Kato T, Shimono Y, Hasegawa M, Jijiwa M, Enomoto A, Asai N, Murakumo Y and Takahashi M: Characterization of the HDAC1 complex that regulates the sensitivity of cancer cells to oxidative stress. Cancer Res 69: 3597-3604, 2009.

29. Ungerstedt JS, Sowa Y, Xu WS, Shao Y, Dokmanovic M, Perez G, Ngo L, Holmgren A, Jiang X and Marks PA: Role of thioredoxin in the response of normal and transformed cells to histone deacetylase inhibitors. Proc Natl Acad Sci USA 102: 673-678, 2005.

30. Yan GR, Xu SH, Tan ZL, Liu L and He QY: Global identification of miR-373-regulated genes in breast cancer by quantitative proteomics. Proteomics 11: 912-920, 2011. 